\title{
Displasia cemento-óssea florida: relato de caso
}

\author{
Florid cemento-osseous dysplasia: case report
}

\section{La displasia cemento ósea florida: reporte de un caso}

\author{
Lucieni Cristina Trovati MORETI ${ }^{1}$ \\ Pedro Augusto Caetano BARBOSA ${ }^{2}$ \\ Nilton César Pezati BOER ${ }^{3}$ \\ Karina Gonzales Câmara FERNANDES ${ }^{4}$ \\ Mitsuru OGATA ${ }^{5}$
}

\author{
${ }^{1}$ Professora da Disciplina de Endodontia, Faculdade de Odontologia da Universidade Camilo Castelo Branco, UNICASTELO, \\ Campus Fernandópolis, Fernandópolis-SP, Brasil \\ Mestre em Radiologia \\ ${ }^{2}$ Cirurgião-Dentista, Graduado pela Faculdade de Odontologia da Universidade Camilo Castelo Branco, UNICASTELO, \\ Campus Fernandópolis, Fernandópolis-SP, Brasil \\ ${ }^{3}$ Professor da Disciplina de Endodontia, Faculdade de Odontologia da Universidade Camilo Castelo Branco, UNICASTELO, \\ Campus Fernandópolis, Fernandópolis-SP, Brasil \\ Doutor em Histologia; \\ ${ }^{4}$ Professora da Disciplina de Endodontia, Faculdade de Odontologia da Universidade Camilo Castelo Branco, UNICASTELO, \\ Campus Fernandópolis, Fernandópolis-SP, Brasil \\ Mestranda em Endodontia; \\ ${ }^{5}$ Professora da Disciplina de Endodontia, Faculdade de Odontologia da Universidade Camilo Castelo Branco, UNICASTELO, \\ Campus Fernandópolis, Fernandópolis-SP, Brasil \\ Doutora em Endodontia
}

\section{Resumo}

Lesões fibro-ósseas benignas são caracterizadas pela substituição do tecido ósseo normal por tecido conjuntivo fibroso celular com conteúdo calcificado variando distribuição e volume. A displasia cemento-óssea florida não tem etiologia definida, prevalecendo em mulheres negras de meia idade a idosas. Pode se expandir bilateralmente, mostra-se restrita aos maxilares e envolve a região periapical dos dentes com vitalidade pulpar. Na maioria dos casos a lesão é descoberta entre os 30 e 50 anos de idade durante exames radiográficos de rotina, pois geralmente é assintomática. O propósito deste trabalho é o relato de caso clínico de mulher negra de 40 anos de idade, que procurou a Clínica Odontológica da Universidade Camilo Castelo Branco (Unicastelo) - Campus de Fernandópolis, para tratamento endodôntico. Ao exame radiográfico inicial verificou-se lesão de aspecto misto na região de periápice do dente 46, mostrando calcificação densa, circunscrita, cercada por halo radiotransparente estreito e ausência de expansão da cortical óssea. A radiografia panorâmica mostrou imagem mista (radiolúcida com área radiopaca) bilateral, em ambas as arcadas, simétrica e não circunscrita. Os dentes se mostravam hígidos, sem aumento de volume na região e com vitalidade. Considerando a presença das lesões em ambas as arcadas, o diagnóstico inicial foi displasia cemento-óssea florida, sendo o mesmo ratificado por meio das características clínicas e radiográficas. Como tratamento nenhum procedimento cirúrgico foi adotado. Foi instituído o acompanhamento clínico e radiográfico. Ressalta-se com esse caso clínico a importância dos exames complementares por imagem no auxilio do diagnóstico das lesões fibro-ósseas que acometem a cavidade bucal e podem não apresentar sintomatologia.

Descritores: Doenças de Desenvolvimento Ósseo; Diagnóstico por Imagem; Doenças Maxilomandibulares.

\begin{abstract}
Benign fibro-osseous lesions are characterized by the replacement of normal bone with a fibrous connective tissue that undergoes mineralization (calcified masses may vary in size and volume). Florid cemento-osseous dysplasia refers to exuberant lesion that involves multiple quadrants of jaw bones and commonly seen in middle aged black females. Florid cemento-osseous dysplasia can expand bilaterally exclusively in the jaw and involves the participation of the periapical region of teeth with pulp vitality. The lesion usually is asymptomatic. In most cases the damage is discovered between 30 and 50 years of age for routine tests X-ray. This paper reports a case of florid cementoosseous dysplasia in a black woman of 40 years old, who sought Unicastelo Dental Clinic for endodontic treatment. Radiographic examination showed sclerotic masses with radiolucent border of lesions in periapex region in both right and left molar region. No surgical intervention was performed. The patient was kept under observation and regular radiological follow up. This case shows the importance of clinico-radiological features in aid of diagnosis of fibro-osseous lesions involving the oral cavity and has no symptoms.
\end{abstract}

Descriptors: Bone Diseases, Developmental; Diagnostic Imaging; Jaw Diseases.

\section{Resumen}

Lesiones fibro-óseos benignos se caracterizan por la sustitución de hueso normal con un tejido conectivo fibroso que se somete a la mineralización (con cantidades variables de sustancia mineral). Displasia cemento ósea florida se refiere a una exuberante lesión que involucra múltiples cuadrantes de huesos de la mandíbula y comúnmente visto en las mujeres negras de mediana edad. Displasia cemento ósea florida puede ampliar de forma bilateral en la mandíbula e implica la participación de la región periapical de los dientes con vitalidad de la pulpa. La lesión suele ser asintomática y é común en pacientes entre 30 y 50 años de edad. En la mayoría de los casos el descubrimiento de la lesión es casual por la imagen de rayos $\mathrm{X}$ cuando las radiografías son hechas por otro motivo. En este trabajo se reporta un caso de displasia cemento ósea florida en una mujer negra de 40 años de edad, que buscó la Clínica Dental Unicastelo para el tratamiento de endodoncia. El examen radiográfico mostró masas escleróticas envueltas por halos radiolúcidos en la región molar derecha e izquierda. No se realizó la intervención quirúrgica. El paciente se mantuvo en observación y regular de seguimiento radiológico. Este caso muestra la importancia de las características clínico-radiológica de ayuda de diagnóstico de las lesiones fibro-óseas que implican la cavidad oral y no tiene síntomas. Descriptores: Enfermedades del Desarrollo Óseo; Diagnóstico por Imagen ; Enfermedades Maxilomandibulares. 


\section{INTRODUÇÃO}

Dentre as diversas lesões intraósseas que acometem os maxilares estão as lesões fibro-ósseas benignas caracterizadas pela substituição do osso normal por tecido conjuntivo fibroso celular com conteúdo calcificado (osso ou cemento), variando distribuição e volume. Estas entidades patológicas, não neoplásicas, formam um aglomerado de lesões intraósseas, sendo que a terminologia fibro-óssea não caracteriza um diagnóstico, somente descreve um processo que inclui doenças do desenvolvimento ósseo $^{1,2}$.

Desta maneira, as displasias cemento-ósseas e a displasia fibrosa constituem de um conjunto de lesões fibro-ósseas displásicas e benignas. Necessitam de um diagnóstico mais específico, em virtude do tratamento variar de nenhum tipo de terapia ao recontorno cirúrgico e até mesmo remoção total da lesão ${ }^{1,3,4}$.

Este grupo de patologias displásicas apresentam comportamento autolimitante, evoluindo de um estágio osteolítico para o osteoblástico, tendo variáveis como a idade, cor, aspecto radiográfico e distribuição das lesões, fator primordial para sua distinção. As displasias cemento-ósseas são as mais frequentes dentre estas lesões fibro-ósseas, e por apresentarem características clínicas e radiográficas marcantes, que limitam as hipóteses diagnósticas, geralmente não há necessidade da realização de biópsia e estudo anatomopatológico, sendo o diagnóstico diferencial embasado no predomínio da fase da lesão: se osteolítica ou osteogênica ${ }^{3,5}$.

Conhecida também como displasia óssea, esta lesão fibro-óssea não neoplásica ocorre no rebordo alveolar, afetando geralmente mulheres adultas ou idosas. A displasia cemeto-óssea tem sido classificada, segundo o grau de comprometimento do osso afetado, em três tipos: cemento-óssea focal, cemento-óssea periapical e cemento-óssea florida. Estas lesões apresentam estreita relação com os ápices dentários, apresentando calcificações nodulares análogas a depósitos de cementos, assintomáticas e não expansivas. E por se originar em regiões próximas ao ligamento periodontal e demonstrar semelhanças histopatológicas com a sua estrutura, alguns autores associam sua origem ao ligamento periodontal. Outros porém, relacionam o seu surgimento a um defeito extraligamentar no osso em remodelação, ativado por fatores locais e correlacionado a um desequilíbrio hormonal $^{1,3}$.

Conhecida como displasia óssea ou fibroma cementossificante a displasia cemento-óssea florida (DCOF) apresenta etiologia não muito bem definida.Mostra-se como um envolvimento multifocal não restrito a região mandibular, podendo estar confinada em um quadrante ou expandindo-se bilateralmente. Nesta patologia, após a substituição do tecido ósseo por tecido conjuntivo fibroso, ocorre a maturação deste, e o trabeculado ósseo se torna espesso com estruturas curvilíneas, sendo que na fase final trabéculas ósseas individuais unem-se e formam massas lobulares ${ }^{3,6,7}$.

Limitada aos ossos maxilares nas regiões periapicais dos dentes com vitalidade pulpar, a displasia cemento-óssea florida, apresenta características clinicas tais como: envolvimento de todos os quadrantes, geralmente não induzindo a expansão óssea; assintomática, podendo se tornar sintomática na presença de infecções secundárias, devido à compressão de próteses mal adaptadas, exodontias, cirurgias periodontais, e até mesmo biópsia, ou seja, qualquer outro motivo que exponha esse tecido. Frequentemente são descobertas em exames radiográficos de rotina ${ }^{7,8}$.

Sua epidemiologia acomete pacientes de gênero feminino, melanoderma, de meia idade às idosas. A bilateralidade e simetria são achados marcantes dessa entidade patológica, podendo em alguns casos ser uma característica familiar, autossômica dominante herdada com expressão fenotípica variada. Considerada uma condição não neoplásica e benigna, seu envolvimento parece não estar relacionado a presença ou ausência de dente, pois podem aparecer em pacientes dentados ou edêntulos $1,9,10,11$.

Identificada por apresentar diversas áreas escleróticas simetricamente distribuídas nos maxilares, com marcante tendência ao envolvimento bilateral, a displasia cemento-óssea florida geralmente envolve regiões contíguas aos dentes e se restringe ao osso alveolar, não sendo incomum encontrar um envolvimento extenso das regiões posteriores dos quatro quadrantes ${ }^{1,9}$.

O objetivo deste trabalho foi descrever um caso clínico de displasia cemento-óssea florida, contemplando seu diagnóstico, forma de tratamento e proservação.

\section{CASO CLÍNICO}

Paciente EMBA, sexo feminino, parda, 40 anos, procurou a Universidade Camilo Castelo Branco para retratamento endodôntico do dente 46. Ao exame radiográfico inicial, verificou-se lesão de aspecto misto na região de periápice do dente 46 , sendo uma lesão radiopaca medindo $10 \mathrm{~mm}$, mostrando calcificação densa circunscrita cercada por um halo radiolúcido em estreita faixa. Observou-se ausência de expansão da cortical óssea na região do dente avaliado, além, de mucosas hígidas. Ao exame clínico constatou-se que o elemento dental estava assintomático. A princípio, a hipótese diagnóstica era de osteíte condensante. A paciente foi encaminhada para a Clínica de Radiologia, para que fossem 
realizadas radiografias periapicais e panorâmica (Figuras 1 e 2).

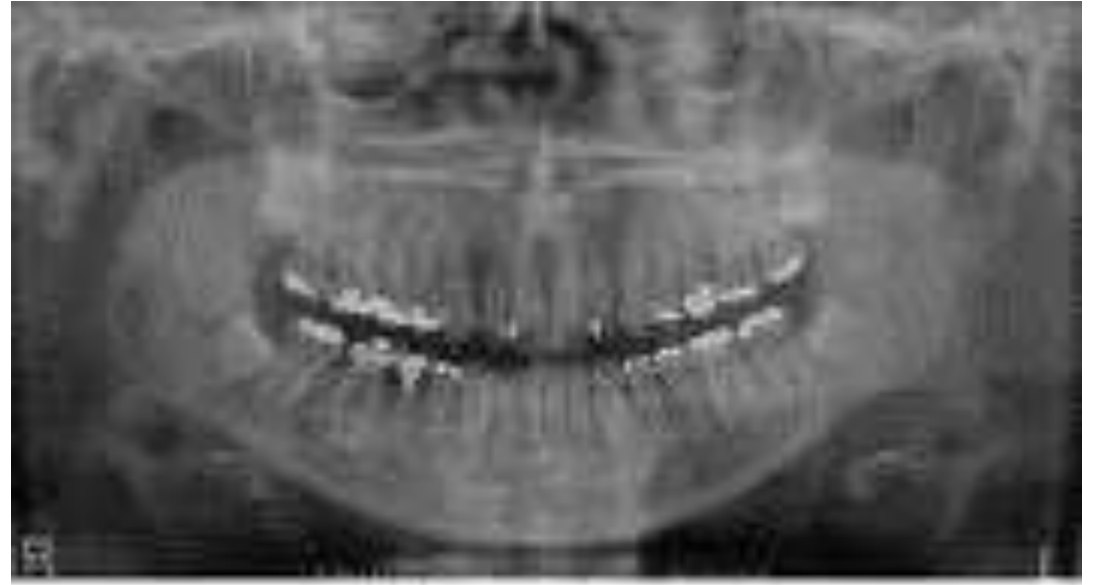

Figura 1. Exame radiográfico panorâmico no qual se nota a presença de áreas radiolúcidas e radiopacas (mista), simétricas e não circunscritas em ambas as arcadas

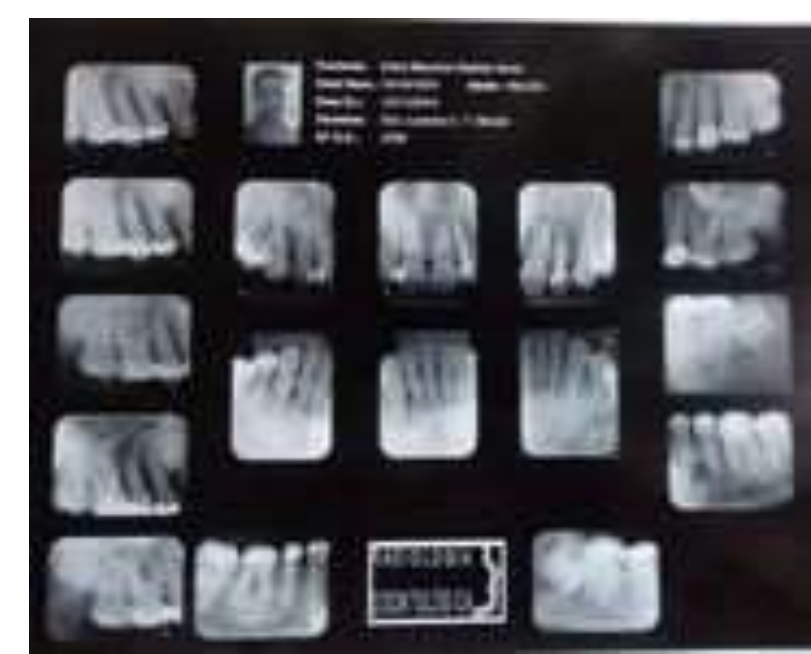

Figura 2. Exame radiográfico periapical completo, no qual são observadas imagens mistas

(radiolúcidas com massas radiopacas) bilaterais

Após a análise dos exames radiográficos foram observadas imagens mistas (radiolúcida com massa radiopaca) bilaterais, tanto na arcada inferior como na superior simétricas e não circunscritas. As principais regiões afetadas eram as dos caninos superiores e incisivos e molares inferiores, sendo que estas regiões apresentaram o espaço do ligamento periodontal e a lâmina dura preservados. Os dentes estavam hígidos, sem aumento de volume na região, sem desajustes oclusais significativos e responderam positivamente aos testes de vitalidade pulpar e sem nenhum outro sinal ou sintoma clínico que justificasse a imagem radiográfica obtida. A mucosa estava integra e não havia expansão óssea.

Considerando a presença das lesões em toda a arcada superior e inferior, o diagnóstico compatível foi displasia cemento-óssea florida. Em virtude dos aspectos clínicos como a raça, idade, ausência de sintomatologia, aspectos radiográficos, além do teste de vitalidade pulpar positivo em todos os dentes, o diagnóstico final foi de displasia cemento-óssea florida. Como o diagnóstico foi obtido pelas características clínicas e radiográficas, não houve necessidade de biópsia, evitando risco de infecção secundária. Como não é indicado tratamento quando esta lesão não mostra sinais de infecção ou de maior agressividade, optou-se por realizar exames radiográficos periódicos (anuais) de controle (Figuras 3 e 4).

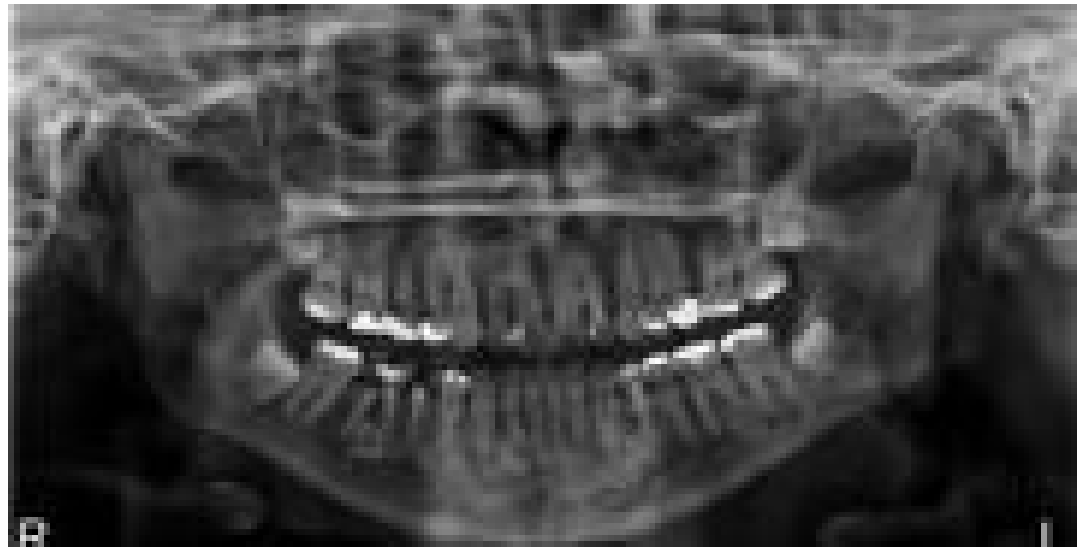

Figura 3. Radiografia panorâmica de proservação (acompanhamento após 1 ano). Não houve mudança no padrão da lesão
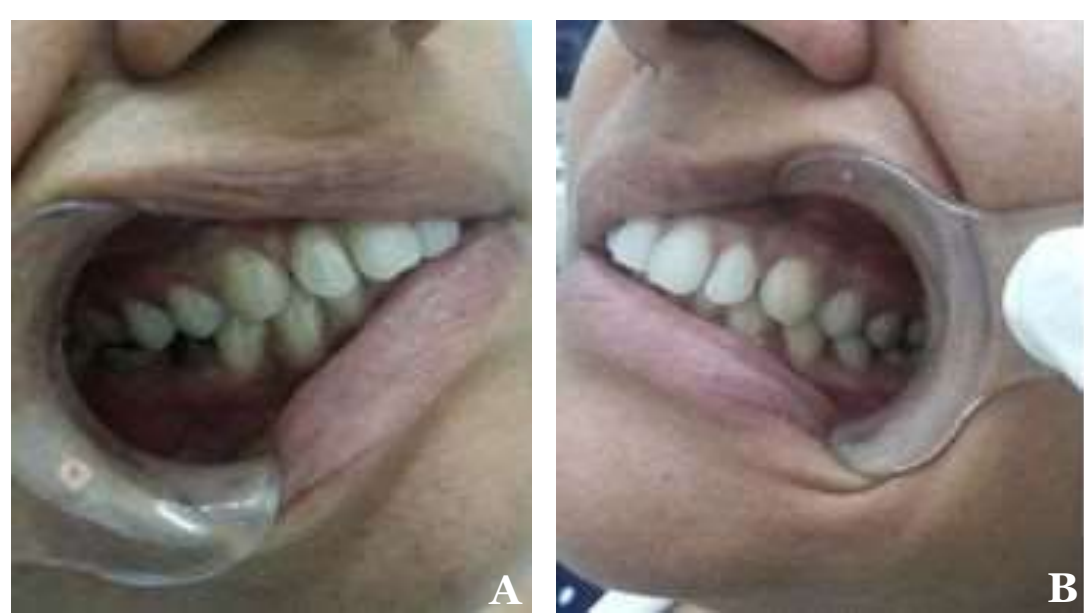

Figuras 4. Aspecto clínico do caso após 1 ano lados direito (A) esquerdo (B). Ausência de coloração anormal da mucosa ou expansão óssea.

\section{DISCUSSÃO}

As lesões fibro-ósseas dos ossos maxilo-faciais constituem um grupo diversificado de condições patológicas. Independentemente do tipo, estas alterações podem apresenta a substituição de osso normal por tecido conjuntivo fibroso com mistura de produto mineralizado, incluindo osteóide, osso maduro e / ou calcificações semelhantes ao cemento. Neste grupo estão inseridas lesões de desenvolvimento, displásicas ou reativas, assim como neoplasias ${ }^{2}$.

Primeiramente descrita por Melrose et al. ${ }^{13}$, a displasia cemento-óssea florida era denominada de lesão displásica ou anomalia de desenvolvimento provenientes de áreas de suporte dos dentes. O termo 'florida' foi proposto por Waldron ${ }^{7}$ em razão da semelhança das densas massas escleróticas de cemento.

A maioria dos autores, de acordo com Monti e França ${ }^{4}$ concordam no que tange à etiologia, sintomatologia e tratamento da displasia cementoóssea florida, e reforçam que ela é uma patologia bem definida dentro do grupo das displasias cemento-ósseas $^{1,3,4}$.

Essa entidade patológica de acordo com a classificação de tumores odontogênicos preconizada pela Organização Mundial da Saúde é considerada uma patologia não neoplásica, constituída por uma proliferação fibroblástica relacionada a trabéculas 
irregulares de osso trançado e massas lobulares densamente escleróticas, compostas de glóbulos de material relativamente acelular semelhante a cemento, dispostos em camadas ou fundidos ${ }^{14,15}$. A análise anatomopatológica de duas lesões removidas após cirurgias citadas por Benjelloun et al. ${ }^{16}$, demonstraram uma massa irregular de cemento e tecido ósseo dentro de um estroma fibroso, sendo este tecido fibroso composto por fibroblastos sem núcleos atípicos e contendo massas calcificadas de cemento, confirmando diagnóstico de displasia cemento-óssea florida.

Caso ocorram infecções secundárias é estabelecido o tratamento conservador à base de antibióticos ${ }^{8}$. Entretanto, se o tratamento medicamentoso não sanar o problema, um tratamento mais invasivo deve ser indicado, conforme salientaram Freitas et al. ${ }^{17}$. Em virtude da lesão ser de natureza avascular, geralmente esta situação complica seu tratamento, induzindo a necrose do osso periapical, com ausência de resposta ao tratamento conservador (antibioticoterapia). Nesse caso tratamento cirúrgico pode ser preconizado, e como consequência, causar um defeito periapical no arco, necessitando de reconstrução com enxertos ósseos ${ }^{1,17}$.

Benjelloun et al. ${ }^{16}$ relataram dois casos clínicos de displasia cemento-óssea florida, sintomáticos, sendo um em virtude da atrofia do osso alveolar e ulceração da mucosa sob a prótese total (primeiro caso) e outro pela infecção pulpar e posterior exsudação extra bucal (segundo caso). Ambos, por apresentaram infecções secundárias, foram submetidos a uma abordagem cirúrgica, com enucleação conservadora da lesão somente nas áreas sintomáticas. No caso clínico relatado neste trabalho não houve sintomatologia dolorosa e muito menos infecção secundária, sendo a paciente orientada a realizar apenas acompanhamentos radiográficos anuais.

Ao contrário de outras lesões que presentes na mandíbula para a displasia cemento-óssea florida não é aconselhável a realização de biopsia, pois eleva as chances de fraturas na mandíbula e infecções secundárias. No entanto, a remoção cirúrgica não é o tratamento proposto para este tipo de lesão. O diagnóstico diferencial com a Síndrome de Paget e com a Síndrome de Gardner deverá ser preconizado ${ }^{18}$. A síndrome de Paget, além de ser uma doença poliostótica, afeta toda mandíbula, ao contrário da displasia cemento-óssea florida que localiza-se acima do canal mandibular. Outra patologia incluída a esse diagnóstico diferencial é a osteomielite esclerosante difusa, porém nesta o dente envolvido não apresenta vitalidade pulpar, além da lesão ser única, pouco delimitada e relacionada a um processo infeccioso ${ }^{18,19}$.

Monti e França ${ }^{4}$ ressaltam que é fundamental que o diagnóstico desta patologia esteja baseado principalmente nos achados clínicos e radiográficos, não havendo necessidade em grande parte dos casos, de biópsias para a sua confirmação. Esta, na medida do possível deve ser evitada, pois procedimentos invasivos nas áreas envolvidas tais como exodontias e biópsia óssea têm sido associadas com a ocorrência de osteomielites. Para o diagnóstico diferencial com a displasia cemento-óssea florida, certas lesões como doença de Paget, osteomielite esclerosante difusa e cementoma gigantiforme familial devem ser consideradas $^{1,4}$, sendo porém excluídas.

Souza Junior et al. ${ }^{15}$ reforçaram que os casos de displasia cemento-óssea florida podem apresentar duas situações clínicas distintas. Na primeira, as massas escleróticas são assintomáticas, não apresentando sinais de inflamação, requerendo apenas proservação com radiografias periódicas. $\mathrm{Na}$ segunda, uma inflamação está presente à condição preexistente, requerendo antibioticoterapia e intervenção cirúrgica. Corroborando com esta citação, o caso clínico descrito foi assintomático, sem sinal de inflamação e ausência de assimetria facial, necessitando somente de acompanhamento clínico e radiográfico . $^{4}$

Frequentemente, de acordo com Bittencourt et al. $^{20}$, em situações típicas, a idade, o sexo, a localização, o aspecto radiográfico e a vitalidade pulpar auxiliam no diagnóstico da condição referida, tornando contra indicada a biópsia. Entretanto, quando estes fatores são atípicos recomenda-se uma biópsia. A maior preocupação é a falha em identificar a natureza da lesão, o que pode resultar em um tratamento endodôntico ou exodôntico desnecessários, por se acreditar que a lesão represente uma lesão periapical inflamatória.

Autores como Melrose et al. ${ }^{13}$ e Neville et al ${ }^{1}$. afirmam que nos casos de lesões assintomáticas os achados radiográficos são casuais ${ }^{21}$, concordando assim com este estudo, já que a paciente tinha como motivo para a realização da tomada radiográfica o retratamento de canais radiculares, os quais se mostravam assintomáticos.

A displasia cemento-óssea florida, frequentemente acomete mulheres negras, na faixa etária dos 40 anos, de causa desconhecida, situação semelhante à encontrada neste estudo, no qual a paciente era sexo feminino, parda, com 41 anos de idade e com as lesões de etiologia ignorada ${ }^{1,4,19}$.

Para Alves e Manzi $^{7}$ a doença pode ser completamente assintomática e, em tais casos, só é descoberta em exame radiográfico de rotina. Em outras circunstâncias, o paciente pode queixar-se de dor intensa, havendo exposição do osso alveolar, avascular, amarelado, na cavidade bucal. Embora raramente seja um achado proeminente, algum grau de expansão pode ser observado em uma ou mais áreas envolvidas. No caso apresentado, em virtude da 
paciente ser assintomática, a escolha foi o acompanhamento clínico-radiográfico realizado anualmente, o que segundo Sarmento et al. ${ }^{22}$ se justifica, pois o tratamento não é necessário, e a melhor opção é o acompanhamento de rotina para a prevenção contra infecção .

Contudo, a etiologia da displasia cemento-óssea ainda é desconhecida ${ }^{9}$. Alterações reativas ou mudanças no ligamento periodontal podem ser evidenciadas no exame histopatológico ${ }^{23}$. Porém há pouca possibilidade de se originar dos tecidos do ligamento periodontal, quando este ligamento periodontal dos dentes relacionados for visível radiograficamente ${ }^{24}$. Estudo de Kawai et al. ${ }^{24}$ aponta que estas lesões não são de origem no ligamento periodontal mas sim, da medula do osso, pois quase todos dentes associados com a lesão apresentam espaços normais do ligamento periodontal.

Com o intuito de se evitar complicações infecciosas ocasionadas por extrações dentárias intempestivas, biópsias e traumas, o tratamento deve ser preventivo, com orientações de higiene bucal entre outras $^{25}$. Em pacientes afetados pela displasia cemento-óssea florida, segundo Dumas et al. ${ }^{26}$ é recomendada profilaxia antibiótica antes do tratamento endodôntico ou qualquer outro atendimento odontológico. No caso em questão, a paciente foi medicada com antibiótico para a realização do retratamento endodôntico.

Bencharit et al. ${ }^{6}$ acrescentam que complicações sérias podem surgir em virtude de irritação pelo uso de próteses sobre áreas desdentadas acometidas com a doença.

No entanto, em razão da natureza avascular da lesão, os antibióticos podem não ser eficazes na infecção ${ }^{9}$, necessitando debridamento cirúrgico ou ressecção apenas na área sintomática, seguido de exame anatomopatológico da amostra ${ }^{24,27}$.

Em áreas assintomáticas está contra indicada qualquer tipo de intervenção cirúrgica, pois a ressecção alveolar deixa um defeito considerável para o nível da borda inferior ${ }^{27}$.

\section{CONCLUSÃO}

A displasia cemento-óssea florida é mais comum em mulheres negras acima de 40 anos de idade, restrita aos ossos maxilares e frequentemente assintomática. Pode apresentar infecção secundária, confundindo-se com quadro clínico de osteomielite primária. Ressalta-se com esse caso clínico a importância dos exames complementares por imagem no auxilio do diagnóstico das lesões fibro-ósseas que acometem a cavidade bucal e podem não apresentar sintomatologia.

\section{REFERÊNCIAS}

1. Neville BW, Damm DD, Alen CM, Bouquot JE. P atologia Oral e Maxilofacial. 2.ed. Rio de Janeiro: Guanabara Koogan; 2004.

2. Grewal HK, Goel P, Batra R, Chopra S . A tender swelling of the left posterior mandible: An unusual case. J Oral Maxillofac Pathol. 2014;18(2):332.

3. Marcucci G. Fundamentos de Odontologia: Estomatologia. Rio de Janeiro: Ed. Guanabara Koogan; 2005.

4. França DCC, Monti LM, Castro AL, Soubhia AMP, Coclete GA, Aguiar SMHCA. Displasia cemento-óssea florida: Relato de caso. Odonto. 2012;20(40):95-100.

5. Quirino MRS. Displasia cemento-óssea florida: relato de um caso. Rev biociênc Taubaté. 2000;6(1):11-14.

6. Bencharit S, Schardt-Saccod D, Zuniga JR, Minsley GE. Surgical and prosthodontics rehabilitation for a patient with aggressive florid cement-osseous dysplasia. J Prosthet Dent. 2003;9(3):220-4.

7. Alves CR, Manzi FR. Displasia cemento-óssea florida: relato de caso clínico. Arq bras odontol 2007;3(1):25-31.

8. Waldron CA. Fibro-osseous lesions of the jaws. J Oral Maxillofac Sug 1993;5(1): 828-35.

9. Beyluoni I, Farge P, Mazoyer JF, Coudert JL. Florid cemento osseus dysplasia: report of case do cumented tomography end 3D imaging. Oral Surg Oral Med Oral Pathol Radiol Endod. 1998;85(6): 707-711.

10. Soares ECS et al. Lesões fibro ósseas: displasia cemento óssea periapical versus Displasia cemento óssea florida. Rev Fac. Odontol Porto Alegre 1998;39(2):26-30.

11. White C, Pharoah MJ. Oral radiology: principles and interpretation. 4 ed. St Louis: Ed. Mosby; 2000.

12. Jerjes W, Banu B, Swinson B, Hopper C. Florid cemento-osseous dysplasia in a young Indian woman. Rev Brit Dent J. 2005;18(8):4778.

13. Melrose RJ, Abrams AM, Mills BG. Florid osseous dysplasia: a clinical-pathologic study of 34 cases. Oral Surg Oral Med Oral Pathol. 1976;41(1):62-82.

14. Sobral APV, Herculano HLT, Balaban R. Avaliação da disposição do íon cálcio em lesões fibro-óssea benignas. Rev Cir Traumatol BucoMaxilo-fac Camaragibe. 2009;9(2):81-8.

15. Souza-Junior EJC de, Menezes ARM, Ramalho LMP, Falcão AFP. Displasia cemento-óessia florida: relato de caso. Odontologia Clin-Cientif. 
2008;7(4):347-5.

16. Benjelloun L, El Harti K, El Wady W. Florid osseous dysplasia: Report of two cases and a review of the literature. Int $\mathrm{J}$ Odontostomat. 2011;5(3):257-66.

17. Freitas DQ, Montebello Filho A, Almeida SM, Bóscolo FN. Displasia cemento-ósse florida: relato de dois casos com diferentes condutas. Rev Min Estomatol 2006;1(5):6-11.

18. Lahbabi M, Fleuridas G, Lockhart R, Delabrouche C, Guilbert F, Bertrand JC. Florid cemento-osseous dysplasia: a poorly recognized entity. Apropos of 5 cases. Rev Stomatol Chir Maxillofac. 1998;99(1):33-9.

19. Gonçaves M, Pispico R, Alves FA, Lugão CEB, Gonçalves A. Clinical radiographic, biochemical and histological finding of florid cemento osseous dysplasia and reporto f a casa. Braz Dent J. 2005;16(1):247-50

20. Bittencourt S, Meira AL, Ferreira PS, Tunes UR, Ribeiro EDP, Casati MZ. Displasia cementária periapical - relato de caso. Rev Inst Ciênc Saúde. 2007;25(3):319-21.

21. Jardim ECG, Santiago Júnior JF, Guastaldi FPS,Magro Filho O, Garcia Júnior IR, Jardim Júnior EG . Displasia cemeto-ossea florida- relato de caso. Rev Odontol Araçatuba 2010;31(2):31-4.

22. Sarmento DJ, Monteiro BV, de Medeiros AM, da Silveira EJ. Severe florid cemento-osseous dysplasia: a case report treated conservatively and literature review. Oral Maxillofac Surg. 2013;17(1):43-6.

23. Coleman H, AQltini M, Kieser J, Nissenbaum M. Familial florid cemento osseous dysplasia. J Dent Assoc S Afr. 1996;51(1):766-70.

24. Kawai T, Hiranuma H, Kishino M, Jikko A, Sakuda M. Cemento-osseous dysplasia of the jaws in 54 Japanese patients: A radiographic study. Oral Surg Oral Med Oral Pathol Oral Radiol Endod. 1999;87(1):107-14

25. Bayi EH, El Harti K, El Wady W. Dysplasie cémentaire périapicale. Inf dente. 2004;86(38):2527-30.

26. Dumas M, Ohanian H, Forest D. La dysplasie cémento osseuse floride. J Dent Québec. 2000;37:97-10.

27. Marx RE, Stern D. Oral and maxillofacial pathology: a rationale for diagnosis and treatment. 1 ed. Quintessence: Publishing; 2003.

\section{CONFLITO DE INTERESSES}

Os autores declaram não haver conflitos de interesse.

\section{AUTOR PARA CORRESPONDÊNCIA}

Lucieni Cristina Trovati Moreti

lucienimoreti@hotmail.com

Submetido em 15/04/2016 Aceito em 26/04/2016 